\title{
A Kinetic Module for Modular Structures Based on Rigid Origami
}

\author{
Alessio Mazzucchi ${ }^{1}$
}

Published online: 2 August 2017

(C) Kim Williams Books, Turin 2017

\begin{abstract}
The increased complexity of geometrical shapes in architecture asks for elaborated fabrication methods, which have to be adapted on a case-to-case basis. Kinetic modular structures can fulfil this task. These structures, by definition, employ a kinetic module, which is a deployable unit composed of hinged rigid bodies. Kinetic modular structures are reusable, adaptable to different forms, easily constructible and, theoretically, they allow the creation of kinematic structures. This paper presents a study to show the potentiality of such an approach, exploiting the properties of rigid origami like the triangular Resch's tessellation.
\end{abstract}

Keywords Modular structure $\cdot$ Kinetic module $\cdot$ Origami $\cdot$ Tessellation · Ron Resch · Parametric design

\section{Introduction}

Origami tessellations can be seen as a continuous transformation of a planar sheet of paper into a more complex folded figure. They are defined as patterns composed by the repetition of the same module or unit cell (Dureisseix 2012). This paper focuses on the study of a single module of the origami to exploit its kinematic properties to conceive modular structures. The aim of this work is to create a workflow to exploit origami tessellation to design modular structures. The resulting structures have the peculiarity of begin composed by a kinetic module that grants to the final structure the possibility of having different final shapes. In the sixties, Ronald Resch, observing how paper crimps, discovered a family of tessellations and throughout his works he focused on how to generate them (Resch 1973, 1970). His studies are filled

Alessio Mazzucchi

Mazzucchi.alessio@outlook.com

$1 \quad 16$ Rue des Goncourt, 75011 Paris, France

Birkhäuser 
with physical models that prove the kinematic capabilities and the possible applications of the family of tessellations he discovered. Resch, together with Christiansen (Resch and Christiansen 1970), created the first tool to simulate rigid folding with computers.

In his work, Giesecke (2004) investigates the possibilities of using origami in architecture and, in particular, he focuses on the possibilities of using the deployable properties of origami. He followed a 'learning by doing' approach, creating a series of physical models to understand the advantages and the pitfall of using origami in architecture. In particular, he pointed out the issue of correctly creating the hinges of the mechanism and the construction difficulties linked with the increase in the scale of the model (Giesecke 2004). More recently, Tachi has provided a solid basis for the study of origami. His various works show how to simulate rigid origami (Tachi 2009), how to transform 3D shapes into foldable origami (Tachi 2013) and how to produce a thick mechanism that behaves like a real origami (Tachi 2011). Additionally, he proposes a workflow to exploit origami in architecture (Tachi 2010).

The RVTR research laboratory (Thün et al. 2012) has created a physical prototype for acoustic purposes based on the Resch tessellation, which is the one used also in this work. This study is relevant because it shows the tessellation's capacity for changing its shape. Felbrich et al. (2014) exploit rigid origami properties to devise a multi-agent system, where independent modules, acting together, fold into the desired shape. Even though the system fails in some areas, like the controlling system of the single modules, this work presents the idea of using the same module to create non-identical shapes. The above-mentioned literature focuses on the study of tessellations, on how to digitally simulate rigid folding, and on how to translate an origami into a thick mechanism. Furthermore, some works exploit these principles to develop practical applications, focusing on the kinematic aspect of the origami. This paper combines and builds on the findings of the existing literature, in particular Tachi (2010, 2011) and Resch (1970), to create a workflow to translate origami into modular structures. Potentially, this workflow is able to translate any rigid origami tessellation into a modular structure.

Rigid origami tessellations are a reliable representation of kinematic modular structures because they represent the ideal kinematic scheme of these structures. The exploitation of origami kinematics in architecture entails four main topics:

- The understanding of the kinematic behaviour of the single module of the tessellation;

- The study of a thick mechanism which preserves the properties of the ideal zero thickness origami, to allow the physical fabrication of the model;

- The shaping of the whole structure, according to the given constraints;

- The understanding of the kinematic behaviour of the entire structure, to keep it fully constrained in the final and in the construction stage.

The aspects related to structural performance are not addressed in this paper since the author will focus on them in a future work.

This work is strongly based on digitally driven tools. Parametric modelling and programming have been used to manage the complexity of the workflow. The first 
one allows the creation of smart models adaptable to the given variables, while the second one is a very powerful tool and it is limited only by the knowledge of its user. It permits the creation of more complex logics compared to the results achievable through parametric modelling, since programming may even simulate the physics of the real world. Sharing thoughts and ideas is one of the best methods to spur innovation, which is why the author has chosen to use, when possible, Open Source tools to carry on this work.

This paper starts by presenting the generic workflow, applicable to any tessellation, created to find the most efficient way to translate origami into modular structures. It will then move to a more in-depth analysis of a modular system based on the Resch tessellation. Then, it will study the kinematic simulation of origami. More precisely, it will focus on the analysis of a single module of the tessellation, and on the tessellation as a whole. In this section, the author will also justify his application choices for modelling the kinematics of the origami. The paper will then enquire into the process of designing thick origami mechanisms through parametric modelling. The paper will then present how to fix a modular structure once the desired shape has been obtained. To evaluate the work, a simple case study has been carried out to demonstrate how to apply the workflow to a real situation. It will be presented towards the end, just before drawing the necessary conclusions.

\section{The Workflow}

Tessellations have an interesting kinematic behaviour which can be exploited to conceive kinematic modular systems. For this reason, there is the need to create a workflow to support the translation of rigid origami tessellations into kinetic modular structures. The key elements of the workflow are: digital tools, Tachi's (2011) thick origami theory and his kinematic theory (2009) and origami tessellations. These four topics are strictly connected and they are at the basis of the workflow (Fig. 1), which is the result of their combination. To explain the logic of the workflow one can use the digital tools, since they are the glue of the process. They are used, in various combinations, to carry on the simulation of the origami, for the creation of the parametric models of the mechanisms, and for the final validation of the structure. For example, the simulation of the single module made with a parametric program combined with the thick origami principle (Tachi 2011) allows a parametric model to be created. This model defines the rigid mechanism and grants its fabrication, a fundamental step to translate paper into architecture. The only action that has not been carried out using digital tools is the study of the static considerations to block the structure. These considerations will be solved through a logical process addressed in "Static and Constructive Consideration". Even though this workflow has been applied only to one tessellation, it is conceived to be used with any kind of rigid tessellation. The following part will deal with the modular system, hence how to build it on top of the tessellation. 


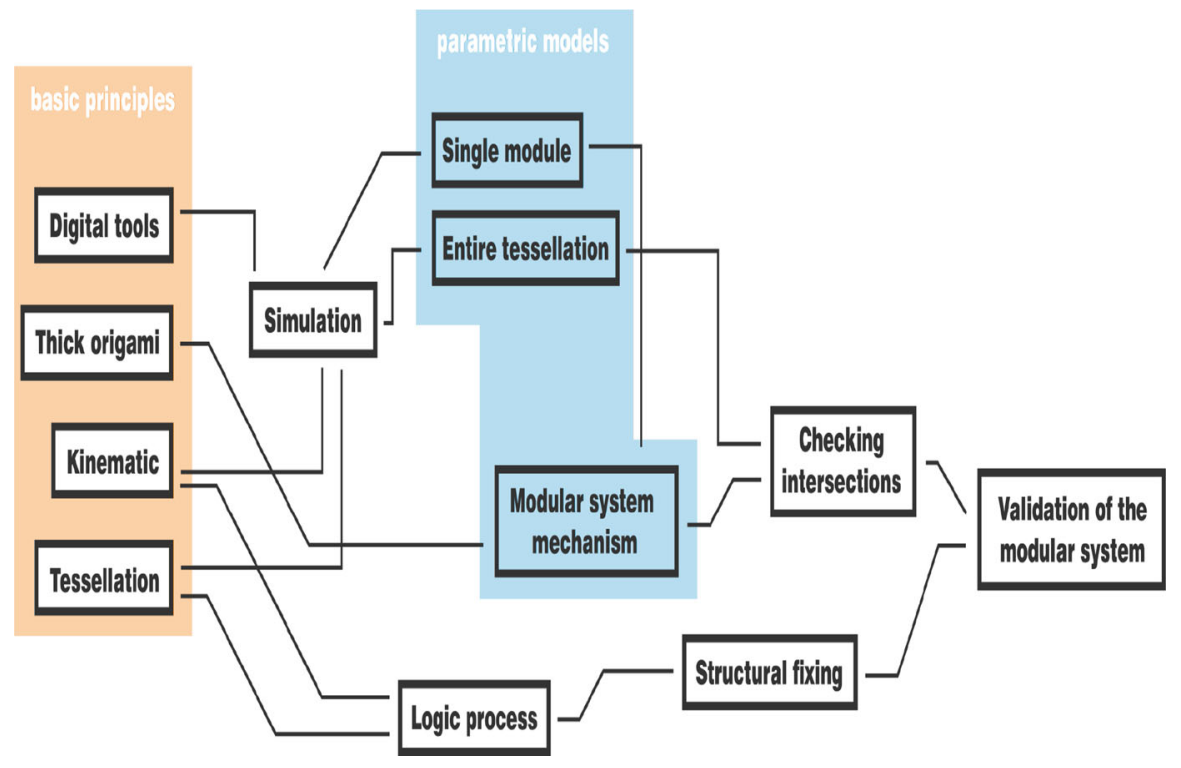

Fig. 1 The diagram of the workflow

\section{The Modular System}

As noted above, origami tessellations are composed of a repetition of one or more unit cells. In his work Freeform Origami Tessellations by Generalizing Resch's Pattern, Tachi (2013) presents a program which, given any 3D geometry, creates a crease pattern of a tessellation. Once folded, the tessellation will approximate the geometry of departure. The only downside is that the unit cells are topologically the same, but their dimensions are different. While showing the interesting feature of the flexibility of this kind of tessellation, this makes it difficult to use for the creation of a modular system, because here we consider a modular system as the repetition of the same module. This led to the understanding that to approximate geometrical shapes one needs a highly adaptable tessellation composed of the repetition of the same unit cell. The triangular Resch's tessellation (Fig. 2) fulfills these two requirements. It is composed of only one module and it can deal with positive and negative Gaussian curvature. Analysing this tessellation, it is possible to identify two elements:

- A big equilateral triangle composed by six right triangles, represented by the grey element in Fig. 3. This element grants the kinematic ability of the tessellation because it is composed of six sub-elements joint together by cylindrical hinges;

- A small equilateral triangle, the orange one in Fig. 3, which is fixed and has the role of connecting all the kinematics elements of the tessellation.

The identification of these two elements is important to translate the ideal tessellation into a rigid thick modular system. To move forward, one needs to 

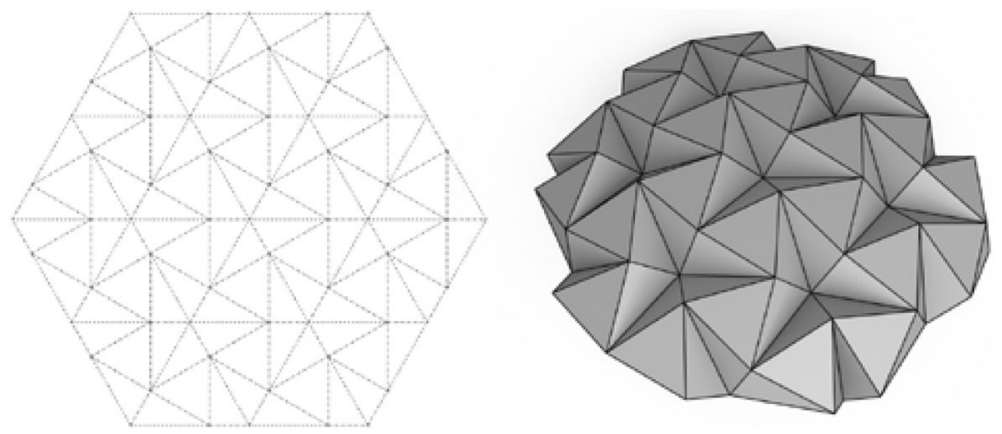

Fig. 2 The pattern and the folded configuration of Resch's triangular tessellation

enunciate the definition of the module used in this work. A module is the kinetic unit element which grants to the modular system the possibility of assuming many shapes. Each time you will read the word module, this is what you should imply. The following sections will investigate how one can design and exploit the ideal properties of the modular system described above.

\section{Kinematic Simulation}

This part will deal with the kinematic simulation of the tessellation by explaining how it has been carried out, and by presenting the programs used. The study of the kinematic of the tessellation has been divided into two separate parts. These parts are independent, since they have different aims that will be explained later on. The first issue analysed has been the study of the mobility of the single module to better understand the dynamics of a single unit. Additionally, it is the best starting point to develop the rigid mechanism, which will be addressed in the following section. On the contrary, the focus of the second point is the folding simulation of the entire tessellation. These two operations have been carried on using different digital tools. In the first step, graph-based parametric programs have been used. Here graph-based refers to the way the constraints imposed on the model are solved (Woodbury 2010). These kinds of programs have a simple interface and they offer an immediate interaction with the model, with interesting features that allow us to experiment and

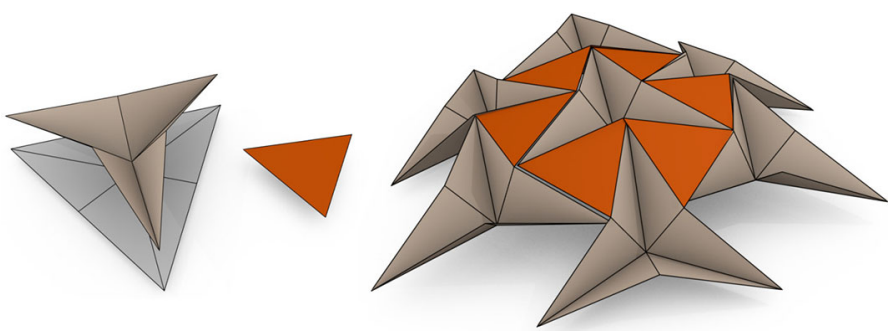

Fig. 3 The two elements that compose the modular system 


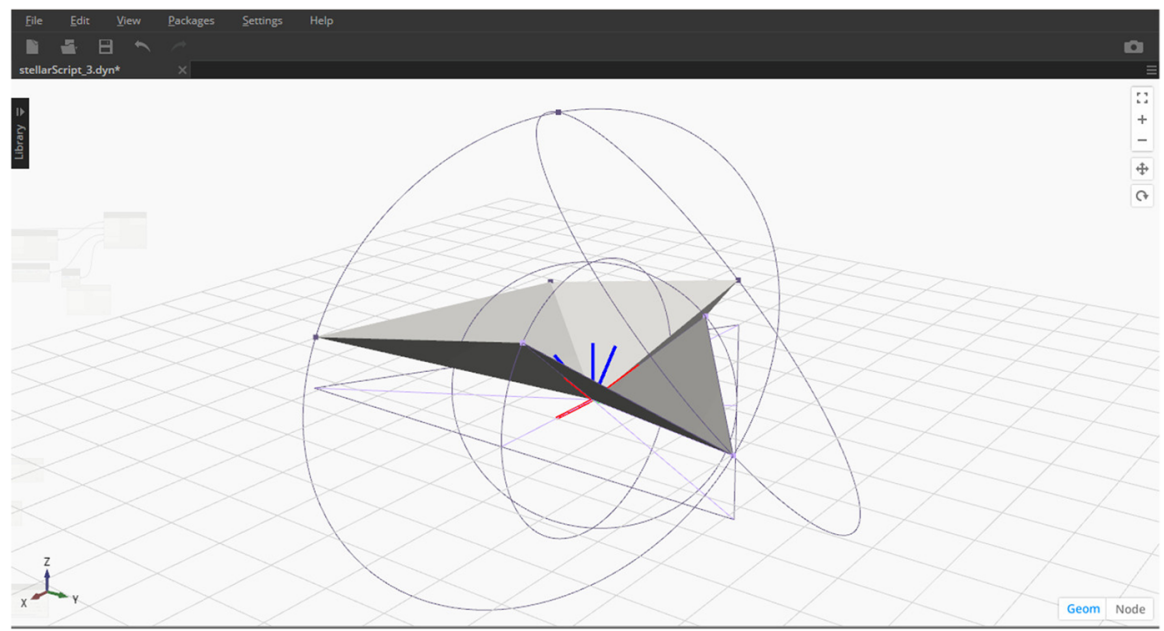

Fig. 4 The simulation of the folding of a single module through constructive geometry principles

create "sketches" of a parametric model. Here Dynamo for Revit has been used because it is directly connected with the BIM environment of Revit and it is open source, even though it is strongly bound with Revit, which is a closed source application. An alternative tool to carry out this task would have been Grasshopper for Rhinoceros. These programs are similar, so the same results could be achieved using one or the other. To carry on the second simulation, the author coded a rigid folding solver using Python and the open source libraries Numpy and Sympy. This simulation could not have been done with graph-based parametric programs because of the way they solve the constraints of the models (Woodbury 2010). Kangaroo, a plug-in for Grasshopper, allows simulation of the folding of origami using a particle physics engine. In order to build an origami model, points substitute for the vertex of the tessellation, whereas springs stand for the folding lines. The main problem of this approach is the instability of the springs and, as a consequence, the risk of losing the isometric transformation of the faces needed to simulate rigid origami. Another alternative solution is the one proposed by Tachi (2009). He created a very powerful and efficient rigid origami simulator (Tachi 2009). Nevertheless, the author decided to build his own solver. Firstly, to better understand the folding mechanism and, secondly, to create custom tools more suitable to morph the tessellation, treating it as though it is composed of modules. This represents a different way of setting the displacement of the tessellation by shifting the focus from the value of every single dihedral angle to the degree of openness of every single module. The following subsection will develop on the study of the mobility of a single module.

\section{The Kinematic of the Single Module}

The behaviour of a single module (Fig. 4) has been implemented in Dynamo, combining node scripting and associative programming (Woodbury 2010), 
calculating the trajectory of the origami vertices using constructive geometry (Casale and Valenti 2012). Through a parametric model the geometrical construction is controlled by the variables, hence the dihedral angles visible in Fig. 5. They represent the internal degrees of freedom of the module. In this way, it is possible to simulate the opening and the closing of the module. The imposition of a symmetry reduces the number of variables of the parametric model. As a result, the movement of the module is controlled by only one parameter. Imposing a symmetry means creating a relation among the three angles, compatible with the movement of the module. One of the possible symmetries is to impose the constraint that all the angles have to be equal. Furthermore, one may decide to add other modules to this parametric model, but this requires finding symmetries not only within the modules, but also among them (Fig. 6). Focusing on the mobility of a single module, one can understand the behaviour of every part of a module and how the parts interact with one another. This single module model is the starting point for studying the fabrication of the thick mechanism presented later on.

\section{The Kinematic of the Rigid Tessellation}

Two main mathematical models of rigid folding exist: the one presented by Tachi (2009) and the other by Schenk and Guest (2011). The main difference between the two lies in the way they consider which are the variables that control the movement of the tessellation. Tachi (2009) focuses on the role of the dihedral angles, while Shenk's model (2011) focuses on the position of the vertexes of the tessellation. The module used in this paper builds on both these works, but it could be considered closer to the second approach. The implementation has been set up considering the vertexes as variables, in line with Shenk and Guest's (2011) findings. Whereas, to integrate and solve the system of equations, Tachi's (2009) method has been used. In this work, the implementation of the mathematical module in Python uses Sympy to set the systems of equations and Numpy to operate with matrices and arrays. The code can be interfaced with both Dynamo and Blender, an Open Source 3D graphic software. To simulate the folding, the system starts by imposing little displacements to every point; subsequently it recalculates the position of every point so that the distance between two points does not change (Schenk and Guest 2011). In this way, we are sure that the kinematic of the origami is respected. A customised function has been created to initialize the tessellation according to the mountain and valley condition of the origami. For further manipulation one may decide to displace a

Fig. 5 The three dihedral angles which control the folding of the tessellation

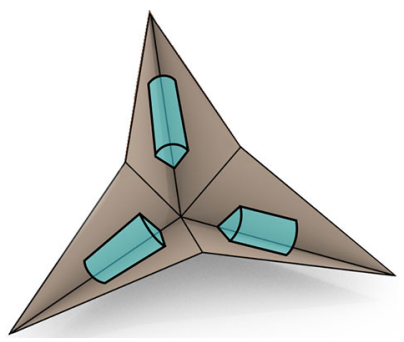




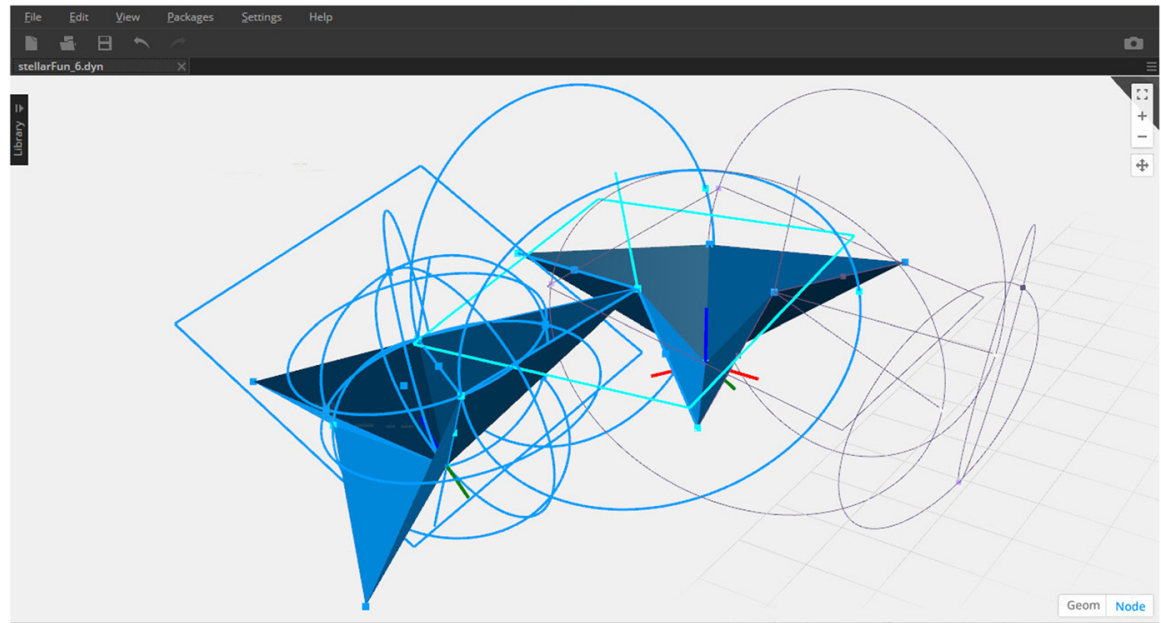

Fig. 6 The simulation of the folding of two modules

single point, a group of them, or to operate on each module of the system by closing or opening it. It has not been possible to directly connect the rigid origami solver with Dynamo due to the incompatibility of Numpy and Sympy with the IronPython implementation of Python in Dynamo. As an alternative, Blender acted as the visualization tool of the simulation (Fig. 7), the resulting file read by Dynamo to obtain, in real time, the tessellation in Dynamo. The next section will present the design of the thick origami mechanism, strongly connected with the kinematic of the single module explained above.

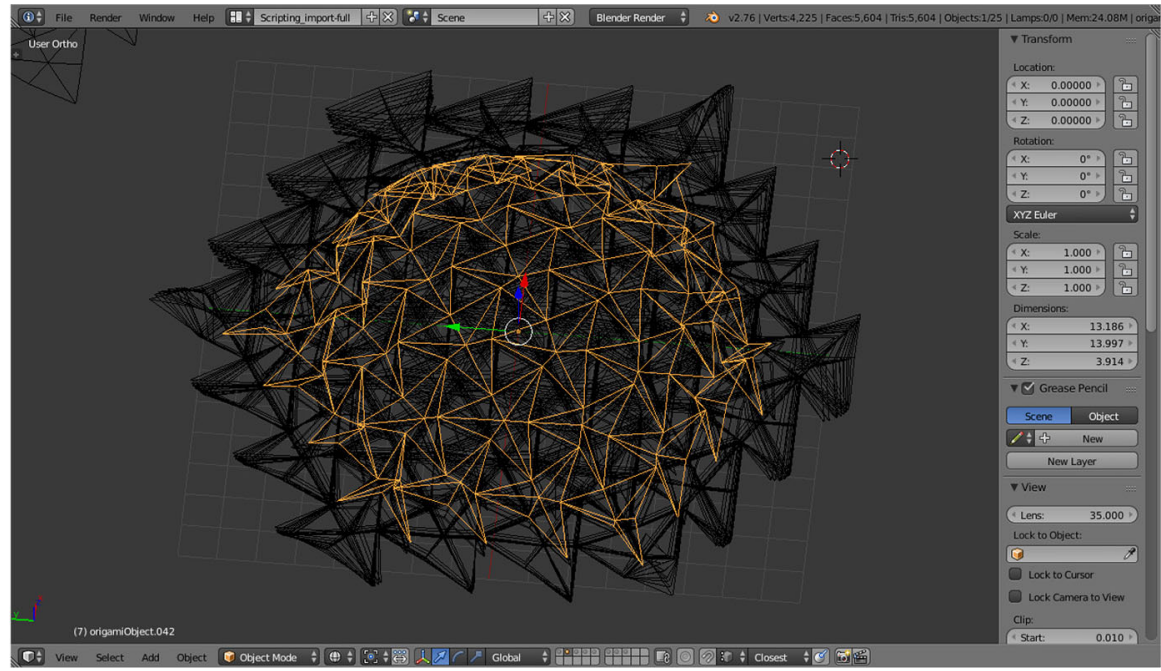

Fig. 7 The rigid simulation performed inside Blender 


\section{Thick Origami Mechanisms}

This section starts by introducing the main problems one faces when designing thick origami mechanisms. Then two different kinds of thick origami mechanisms will be presented and a comparison between the two will mark the end of this section. When building a modular system based on origami principles one must preserve the kinematic properties of origami while increasing their thickness. To grant stiffness to the module one must add thickness to assure its structural performance (Tachi 2011). Tachi (2011) analyses and shows how to create a thick origami mechanism preserving the original kinematic of the origami. The main condition to follow is that the cylindrical hinges of the thick origami must be kept in the same position as the edges of the ideal origami, as explained above. An inevitable downside of adding thickness to an origami is that the module partially loses its ability to fold itself completely. The more thickness we add to a module, the less foldable it is going to be. To limit this effect, it is possible to change the geometry of the mechanism, but in this way the number of variables to define the geometry of the module increase. Two kinds of thick mechanism will be proposed and, as a consequence, each of them will also have their own parametric model. Having a parametric definition of the mechanism assures better control of the geometric variables. This allows a better understanding of which is the best combination of variables to obtain a module with the required thickness and the desired folding ability.

At first the mechanism has been developed by adopting a modified version of the "tapered panels" method proposed by Tachi (2011). Referring to the differentiation of the parts of the single module proposed in "The Modular System", this first mechanism is made of two parts. One part grants the movability of the module, while the other one connects the kinematic elements. To generate the panel of the rigid mechanism, the parametric model extrudes the edges of the module according to the value of the mountain or valley sign of the crease (Fig. 8). This process generates a non-planar panel that is controlled by the maximum foldable angle and by the thickness of the panels (Fig. 9). For a given thickness it is possible to increase the module's folding capability by augmenting the distance of the panel from the vertex. Figure 10 shows how the parameters of the model-six of them, from $p 1$ to $p 6$ - control the distance of the panels from the vertex. To fabricate such a model, the panels are planarized and then their development has been cut with a laser cutter, folded and assembled using adhesive tape (Fig. 11). This paper proposes

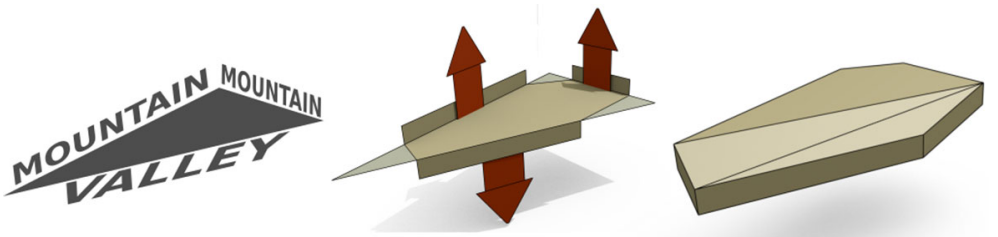

Fig. 8 The process, which shows the steps needed to generate the panels that compose the rigid mechanism 

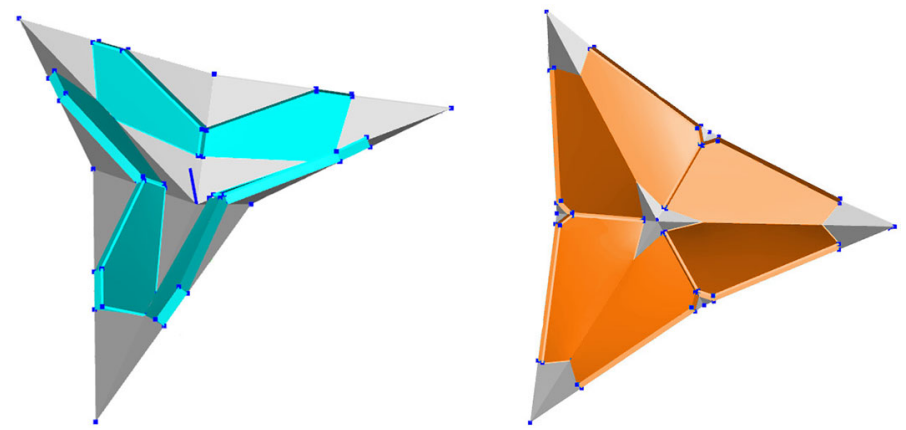

Fig. 9 The image shows two possible configurations of the same rigid mechanism. The main difference between the two versions is their capability to fold. The blue one can fold more because the thick parts are more distant from the vertices of the ideal origami (colour figure online)

Fig. 10 The parameters which control the parametric model of the rigid panel mechanism

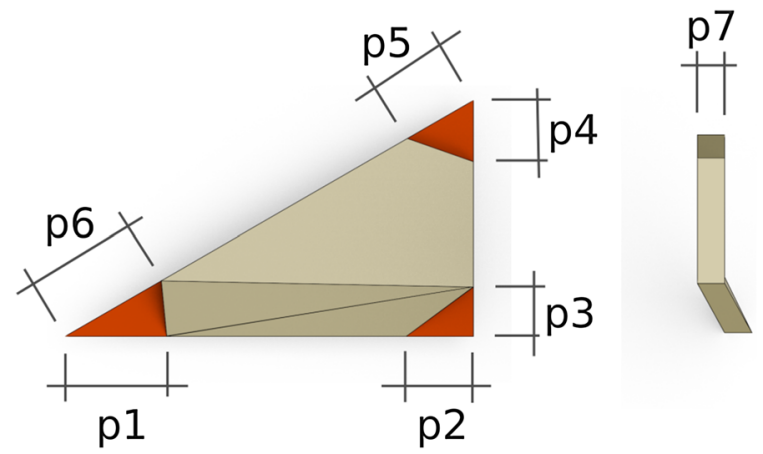

Fig. 11 The physical prototype of the mechanism based on nonplanar panels

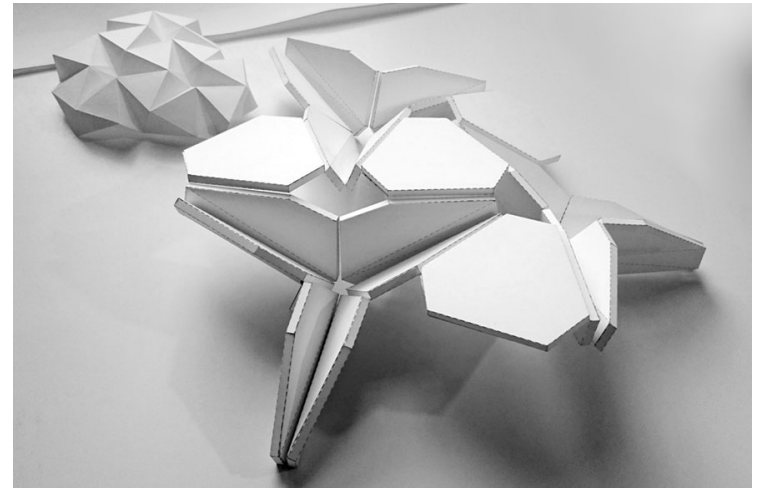

another solution to building the thick mechanism. This second option is based on a pin-joint configuration of the tessellation, losing the idea of origami as unique surface. Since the pin-joint structure is already aligned with the ideal origami, this thick mechanism entails the resolution of only one problem: the creation of a spherical hinge connecting the six converging members. The solution lies in 


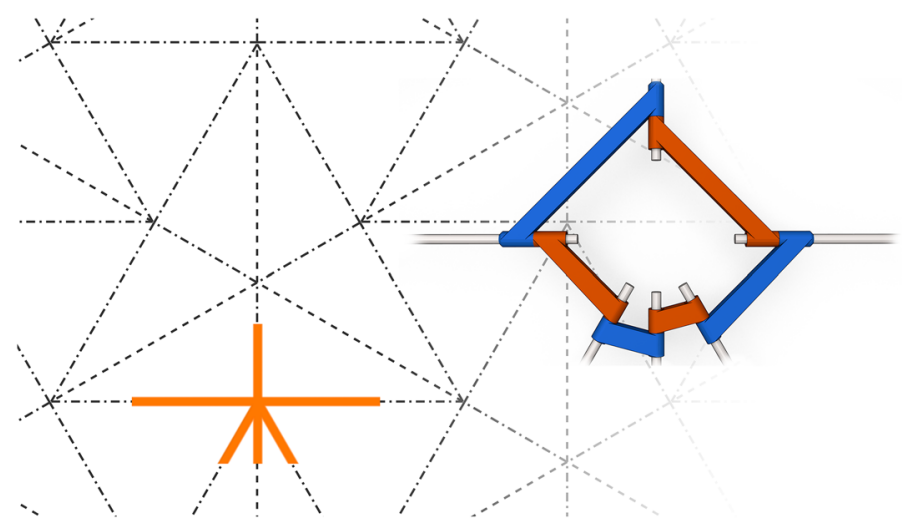

Fig. 12 One of the two connection types that substitute for the spherical hinges

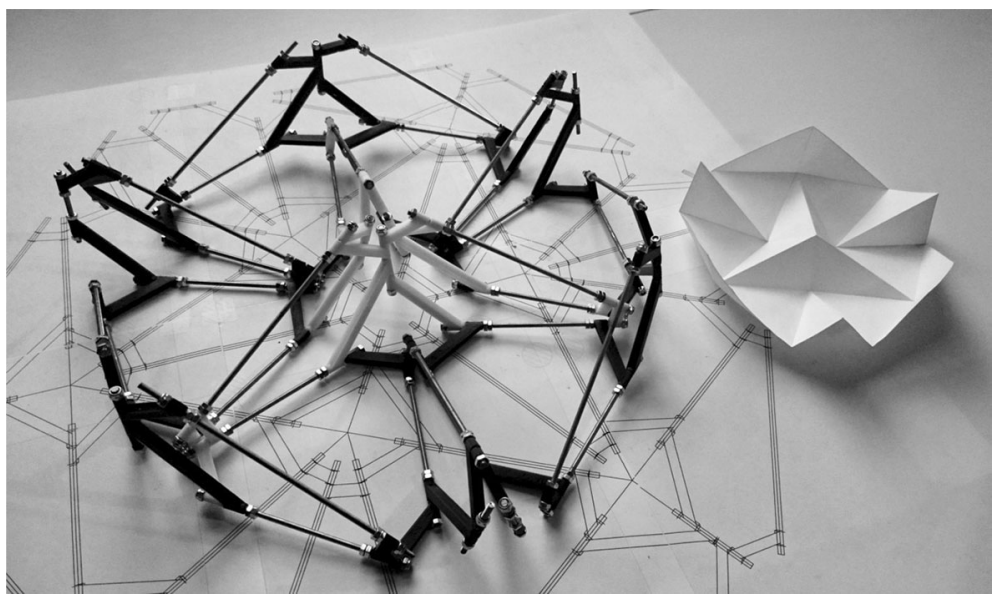

Fig. 13 The physical prototype of the pin-joint mechanism

replacing the spherical hinge with six new joints, each composed of two cylindrical joints connected like a rigid body (Fig. 12). The parametric model of this mechanism has three main constraints that govern the geometrical shape of the joints: the physical dimension of the joints, the maximum folding angle and the need to avoid self-collision among the joints connected to the same member (Fig. 14). As for the model composed of panels, if one wants to augment the thickness of the module without losing folding capability, one is obliged to distance the joints from the vertex. For this second kind of model, a small prototype has been built. Threaded rods are used as substitutes for the members, and the hinges are printed in plastic in 3D (Fig. 13). The two thick implementations of the module presented here are both functional. The one made of panels brings about, when referring to largescale modules, fabrication problems due to the non-planarity of the panels. Whereas the second implementation entails fewer folding issues, although the torsional forces that the joints connecting the members have to face remain unknown. This could 


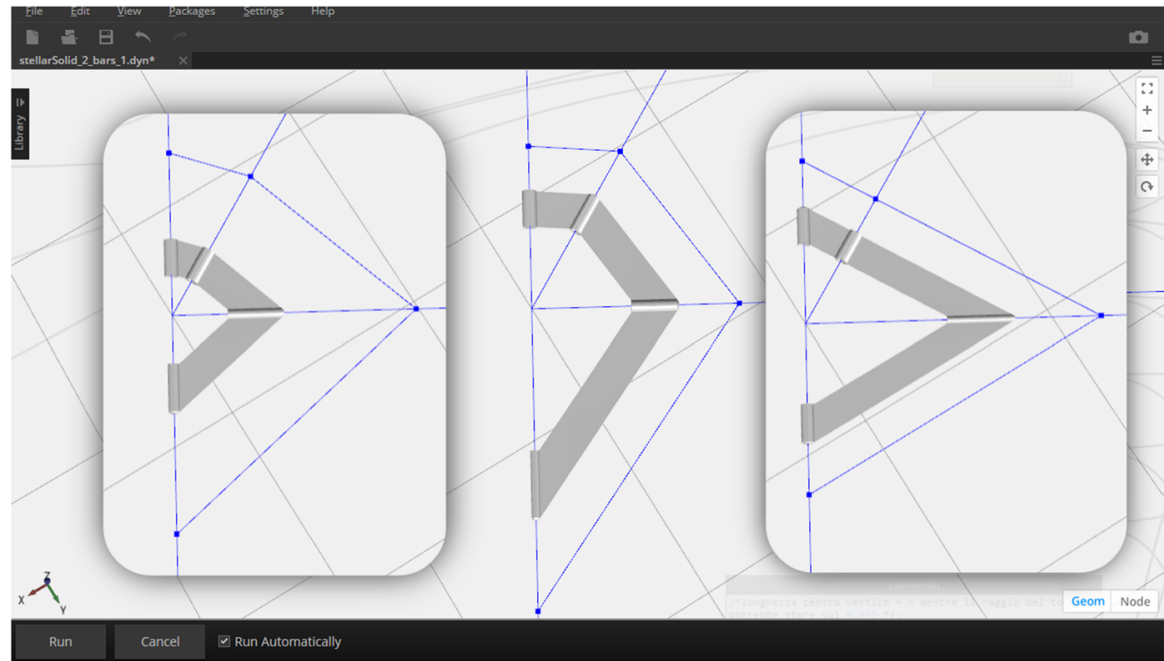

Fig. 14 In the image it is possible to see how the shape of the joints can change, maintaining their alignment with the axis of the origami

Fig. 15 The image shows what happens around a module when it is fixed. The orange part of the tessellation is fixed while the blue part is blocked because of the orange (colour figure online)

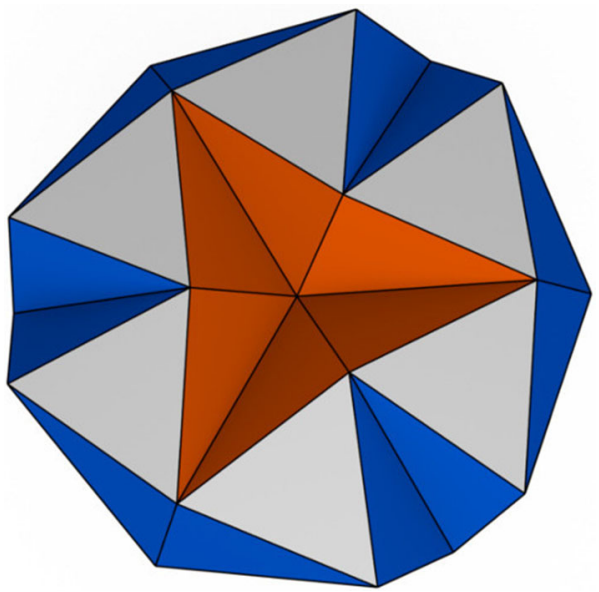

represent an important constraint when choosing the dimensions of the joint, impacting on the maximum foldability. The next section deals with static and constructive considerations on the tessellation.

\section{Static and Constructive Consideration}

When working with a structure based on origami tessellation that, for its nature, is under-constrained, it is essential to establish a strategy to control its kinematic freedom. The easiest method to fulfil this task will be fixing the boundaries of the origami tessellation to fully constrain it. This rule was already known by Resch 
Fig. 16 The image shows that to block one module by propagation it has to be surrounded by three fixed modules
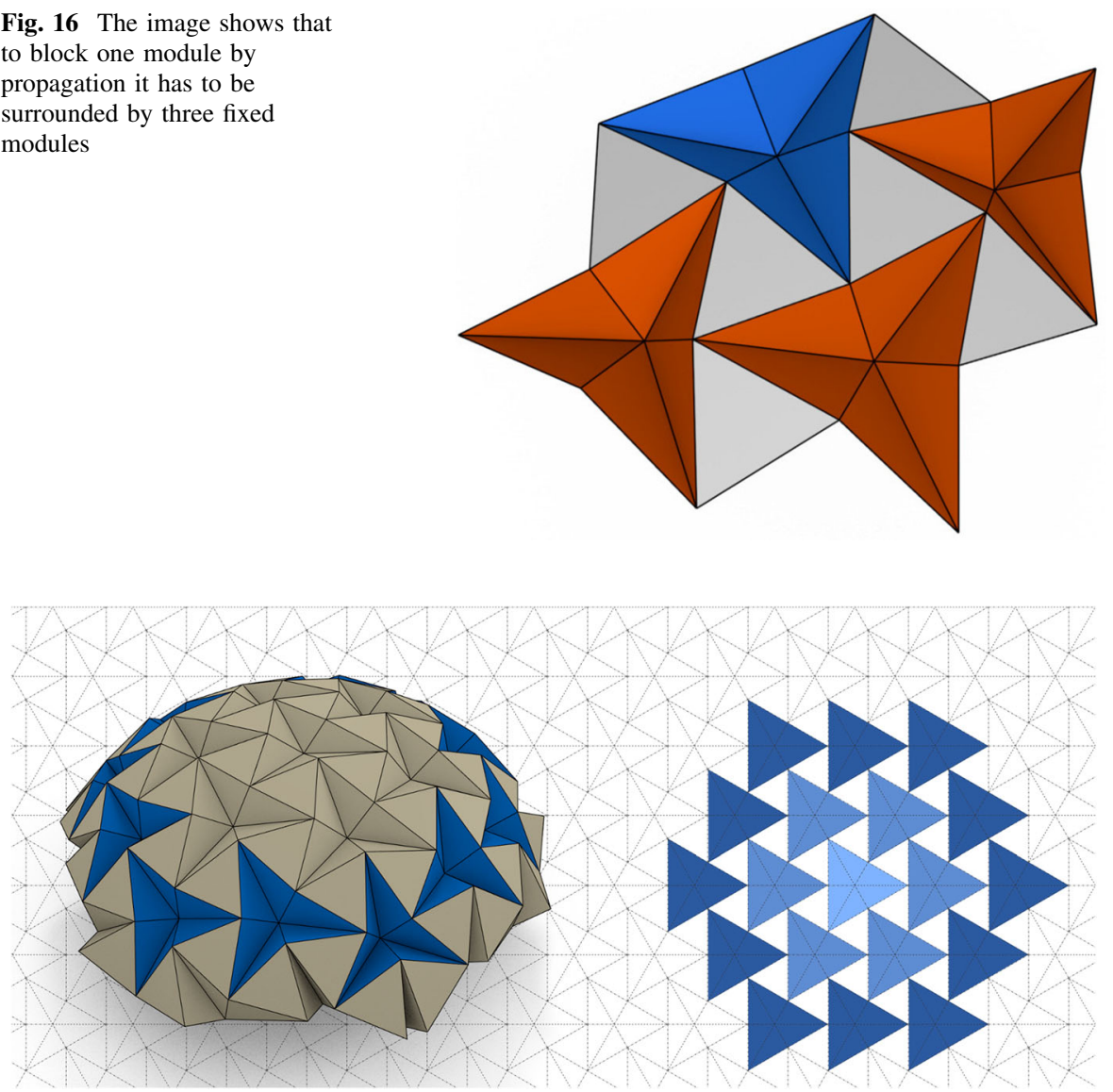

Fig. 17 The kinematic pattern and its folded configuration. The darker modules are the only ones fixed and are positioned all around the boundary

(1970), and it has been mathematically proven by Tachi (2010). From this statement one can deduce that once the perimeter of the tessellation is blocked, one is sure that the entire tessellation is completely fixed. Imagining a real scenario, a dome built following this kind of principle may need some openings and so this kind of structure will not allow the entire perimeter to be anchored to the ground, hence the need to find an alternative way of proceeding. The method proposed in this paper shifts the attention from the whole tessellation to the single module. To come to this solution one needs to analyse two different situations: first of all, what happens around a module when we block it, and secondly what has to happen around a module to block it. The first situation is shown in Fig. 15. The orange module is fixed and, as a consequence, so are the blue parts around it. The second case is illustrated by Fig. 16, where the blue module represents a non-fixed module. Due to the fact that it is surrounded by three blocked modules, represented in the picture by the orange triangles, one can deduce that the blue module is also fixed. Therefore, 


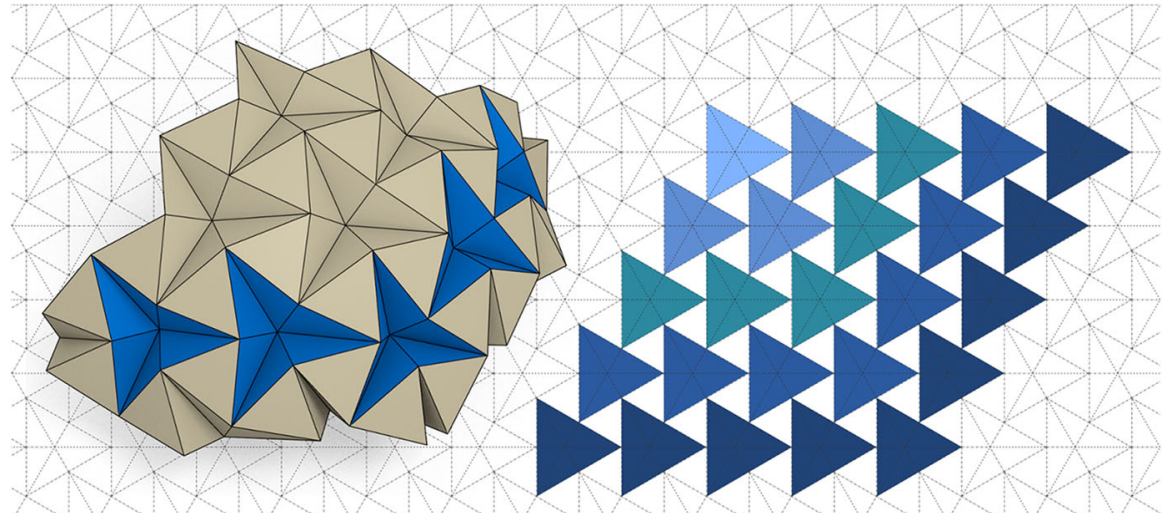

Fig. 18 The kinematic pattern and its folded configuration. The darker modules are the only ones fixed and are positioned on two sides of the boundary, leaving one side free from constraints

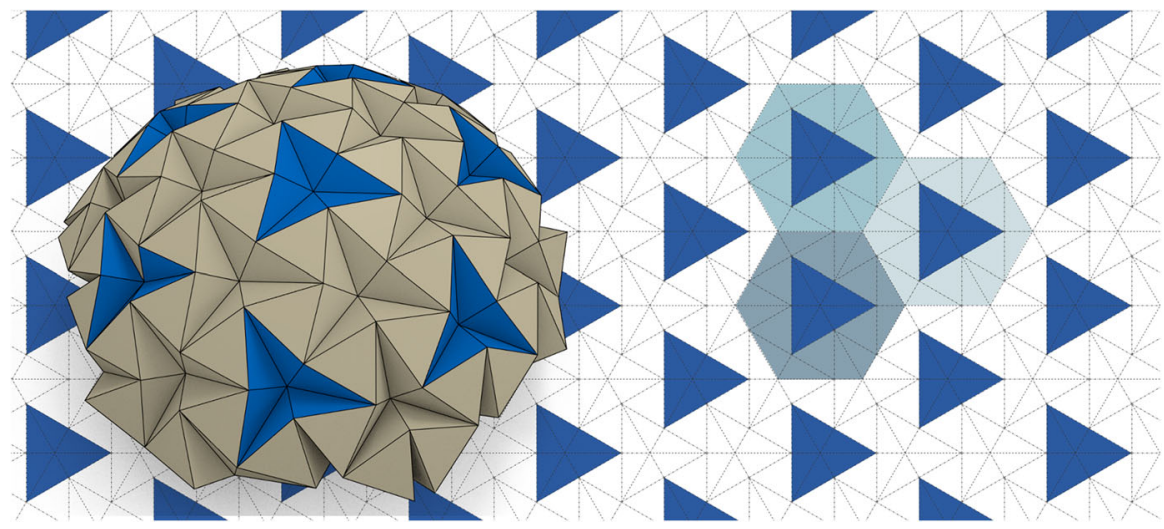

Fig. 19 The kinematic pattern and its folded configuration. The darker modules are the only ones fixed, and they are spread uniformly on the tessellation

putting together these two statements, it is possible to create a visual-logic process to limit the movability of the tessellation to help understand and control the kinematic of it. To prove the feasibility of this process, three different cases will be presented. The first one, Fig. 17, shows that, when blocking all the modules on the perimeter-the darker figures in the picture-one blocks the entire tessellation. A peculiarity of this configuration that differentiates it from the method proposed by Resch is that the blocked modules do not need to touch the ground and the tessellation can be considered internally stable. Figure 18 displays a case where only two sides of the tessellation are blocked, represented by the dark blue triangles, and it is a modified version of the previous case. Figure 19, shows how one can arrange the fixed modules, the blue triangles, in an even way. This could be useful to spread the fixing forces throughout the entire structure, or it can be used as a starting point to decide where to put servo controllers to create a kinematic structure. These methods also suggest how it could be possible to build the structure module by 


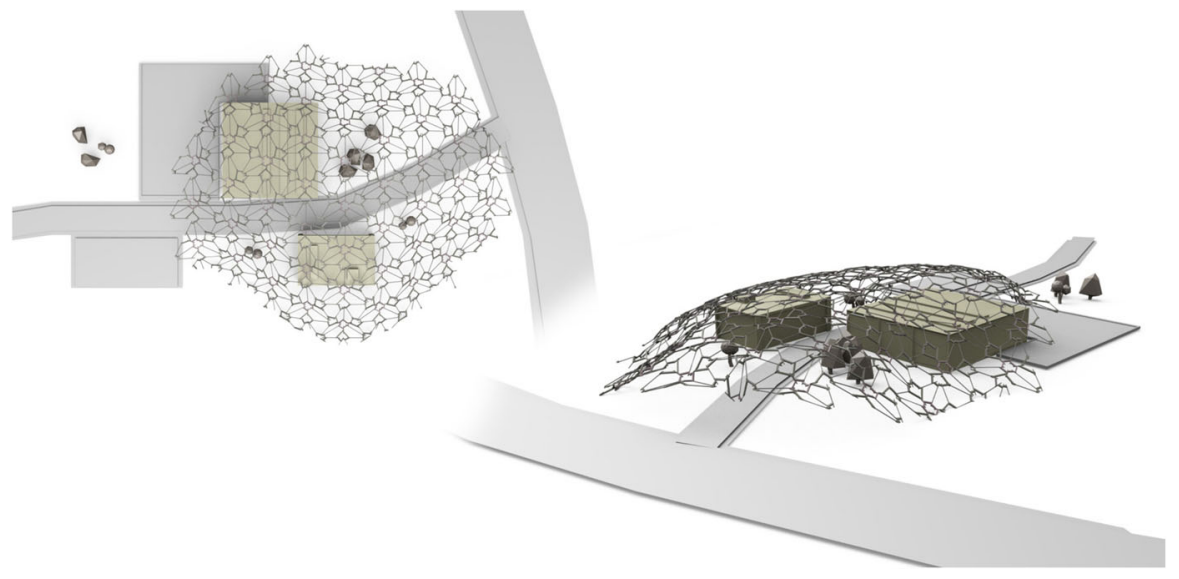

Fig. 20 The dome built using the modular system based on a Resch's tessellation

module, starting from the boundary and without using scaffolding. After dealing with this topic theoretically, the next section will present a case study.

\section{Sandbox}

A fictional and simple landscape has been conceived to test the designing tools and to simulate the placement of the rigid mechanism. The outcome is a dome, based on Resch's tessellation, which covers all the building volumes and grants pedestrian access through the path in the middle of the area (Fig. 20). The dome is a temporary covering for an open area, and its dimensions have been arbitrarily decided by the author, since the aim of the case study is to prove the feasibility of the process described in the previous sections. The first thing to do is to choose which type of tessellation one wants to use. Here, the choice has fallen on the triangular Resch tessellation as explained in "The Modular System". The dimensions of the module and of the tessellation have been adapted to the surface of the site. The dimension of the tessellation has to be bigger than the area it will cover, since it gets smaller when it is folded. Afterwards, the pin-joint mechanism, presented in the section above, has been chosen as the rigid mechanism to use to build the structure (Fig. 21). So, the maximum foldable angle of the mechanism has been obtained. During this phase, it would have been possible to carry on some structural performance analysis to identify the best possible shape for the mechanism. Due to limited timing, this analysis has not been executed in this paper. At this stage, all the parameters needed to carry on the simulation of the ideal tessellation are available and they are: the choice of the tessellation, the characteristics of the rigid module, and the site's constraints. To launch the simulation, the first step is the most critical one. The tessellation in its planar configuration is unstable and needs to be initialized by folding every crease in the right direction, as when folding a paper origami tessellation. Afterwards, the simulation of the folding of the tessellation can start by 
Fig. 21 The module of the Resch's tessellation in its thick version based on the pin-joint solution explain in the section "Thick Origami Mechanisms" above
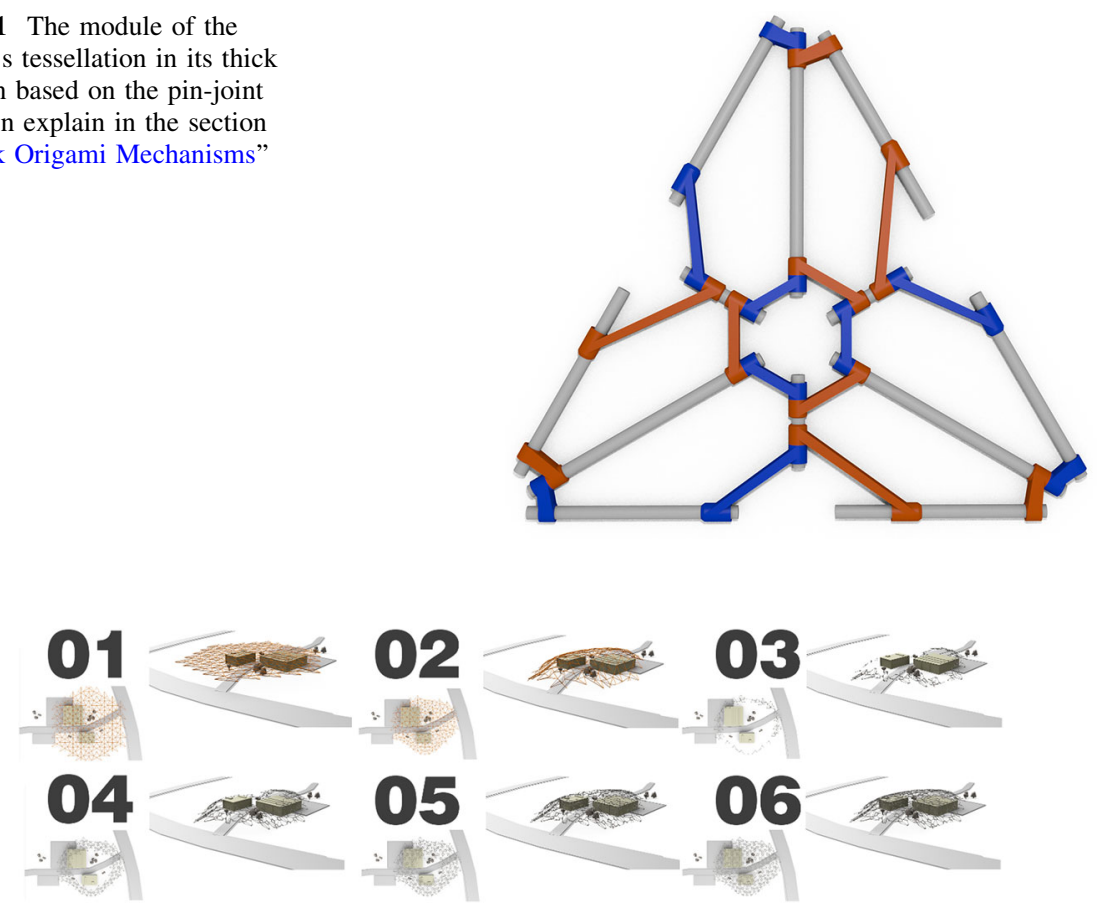

Fig. 22 The sequence show the initialization of the tessellation, its folding, and the steps for assembly of the dome

shifting or fixing its vertexes or by deciding which module to close and which one to open. One can also code new functions to add modules on the boundary of the tessellation or ideate new tools to modify the shape of the tessellation. Since the shape of the tessellation is now clear, the thick mechanism can be applied to it and in this way one can verify that there are no self-intersections among the modules to prove the feasibility of the structure. Using the logical process, explained in the previous section, we can decide which modules to fix to block the structure and to propose the assembling sequence of the modules (Fig. 22).

\section{Conclusion}

This paper fulfilled its aim of creating a workflow to exploit origami tessellation to create a kinematic modular system, handling the main challenges one may face. The key steps of this work are the modelling of the structure based on origami, the research of a fabrication method, and the understanding of the basic kinematic of the structure required to effectively stiffen the structure. Parametric tools have been used to solve the above-mentioned problems, as well as to coordinate all the various aspects of the project. These tools are mainly open source and some of them, like the rigid origami simulator, have been coded by the author using Tachi's (2009) and Schenk's (2011) works as inspiration. As every work, this paper has a number of 
shortcomings. The developed tools are not user-friendly, and so a user interface should be added. The work lacks the structural analysis of the single module and of the entire structure-two important variables that must be included in the workflow since they could influence the final geometry of the single module and of the tessellation as a whole. The study of the details of the structure, like the anchoring on the ground, should be explored. These are very important cues that the author hopes to tackle in upcoming research.

\section{References}

Casale, A. and G. M. Valenti. 2012. Nuovi quaderni di applicazioni di geometria descrittiva. In: Architettura delle Superfici Piegate. Vol 6. Edizioni Kappa. Contributo di Michele Calvano.

Dureisseix, D. 2012. An overview of mechanisms and patterns with origami. International Journal of Space Structures 27(1):1-14.

Felbrich, B., D. Lordick, J. R. Noennig, and S. Wiesenhuetter. 2014. Deployable folded plate structures. In: Proceedings of 16th International Conference on Geometry and Graphics (ICGG), Innsbruck (Austria), August 4-8, 2014.

Giesecke, K. 2004. Deployable Structures Inspired by the Origami Art. Master's thesis, MIT.

Resch, R. D. 1970. The Ron Resch Paper and Stick Film. http://www.ronresch.org/ronresch/gallery/ paper-and-stick-film/ (Accessed 12 June 2017).

Resch, R. D. 1973. The Topological Design of Sculptural and Architectural Systems. In Proceedings of the 1973 National Computer Conference, AFIPS '73, Vol. 42, 643-650.

Resch, R. D. and H. Christiansen. 1970. Design and Analysis of Kinematic Folded Plate Systems. In: Proceedings International Shell Association, Vienna, Austria, September 1970.

Schenk, M. and S. D. Guest, 2011. Origami folding: A structural engineering approach. In: Origami 5: Fifth International Meeting of Origami Science, Mathematics, and Education (5OSME), eds. Patsy Wang-Iverson, Robert J. Lang, and Mark Yim, 293-305. Florida: CRC Press.

Tachi, T. 2009. Simulation of Rigid Origami. In: Origami 4: Fourth International Meeting of Origami Science, Mathematics, and Education, ed. Robert J. Lang, 175-188. Florida: CRC Press.

Tachi, T. 2010. Geometric Considerations for the Design of Rigid Origami Structures. In Proceedings of the International Association for Shell and Spatial Structures (IASS) Symposium 2010, Shanghai, China, 8-12 November 2010.

Tachi, T. 2011. Rigid-foldable Thick Origami. In: Origami 5: Fifth International Meeting of Origami Science, Mathematics, and Education (5OSME), eds. Patsy Wang-Iverson, Robert J. Lang, and Mark Yim, 253-263. Florida: CRC Press.

Tachi, T. 2013. Freeform Origami Tessellations by Generalizing Resch's Patterns. In Proceedings of the ASME 2013 International Design Engineering Technical Conferences and Computers and Information in Engineering Conference (IDETC/CIE 2013), Portland, Oregon, 4-7 August 2013.

Thün, G., K. Velikov, C. Ripley, L. Sauvé, and W. McGee. 2012. Soundspheres: Resonant Chamber. In: Proceedings, ACM SIGGRAPH 2012 Art Gallery, 348-357. Los Angeles, California, 5-9 August 2012.

Woodbury, R. 2010. Elements of Parametric Design. Abingdon: Routledge.

\footnotetext{
Alessio Mazzucchi graduated in engineering and architecture in Italy, where he developed a strong passion for digital tools. After graduation, he moved to France to become a researcher at the MAPCNRS working on point-cloud reconstruction. At the same time, he started an ongoing collaboration with Professor Hemmerling at the Politecnico di Milano. Currently he works as a computational designer and he carries on his personal research on origami, curve folding and new design techniques.
} 